\title{
The Quaternion Structure of Space-Time and Arrow of Time
}

\author{
Yingqiu Gu \\ Fudan University, Shanghai, China \\ Email: yqgu@fudan.edu.cn
}

Received April 6, 2012; revised May 8, 2012; accepted June 1, 2012

\begin{abstract}
In fundamental theories of physics, the dynamical equations all have time inversion invariance. Except for the evolution of some simple system which has realistic inverse processes, but for a slightly more complicated system, the evolution processes are irreversible. This is the problem of arrow of time, which is always warmly debated. In different point of view, we find there may have some conceptual misunderstanding in the controversy: 1) The realization of an inverse process does not mean the time of the system goes backward;2) The principles of relativity and covariance are the constraints to physical laws, but not constraints to specific solutions. The equations must be covariant, but the solutions are not definitely symmetric; 3 ) Time is a global property of the universe, which is a measurement of the evolution process of the universe. The internal time of a matter system reflecting its internal evolution speed also takes this cosmic time as a unified background and standard of measurement; 4) The universe has a unified cosmic time $T$ and a cosmic space related to this cosmic time. They are objective and absolute; 5) The eigensolution of a spinor is a critical state losing time concept, which responses the interaction of environment with some uncertainty, then the evolution process of the world is not uniquely determined; 6) The non-uniqueness of the evolution process means that the inverse process is absent. So for a world including spinors, the evolution is essentially irreversible. In this paper, according to the widely accepted principles and direct calculations of transformation, we reveal the misunderstandings in the usual controversy, and then give more natural and reasonable explanations for structure of space-time and arrow of time.
\end{abstract}

Keywords: Arrow of Time; Invariance; The Principle of Relativity; Quaternion Structure of Space-Time; Simultaneity

\section{Introduction}

In fundamental theories of physics such as Newtonian mechanics, electrodynamics, quantum mechanics, the dynamical equations all have time inversion invariance. But except for the evolution of some simple system which has realistic inverse processes, such as swing of a pendulum, dissolution and crystallization of crystal, emission and absorption of photons, for a slightly more complicated system, the evolution processes are essentially irreversible. This is the problem of arrow of time, which is always warmly debated [1-9].

In different point of view, we find there may have some conceptual misunderstanding in the controversy:

1) The realization of an inversion process does not mean that time of the system goes backward. We make two clocks with mirror image. One rotates clockwise, and the other rotates anticlockwise. But this does not mean time of the clockwise one goes forward, and time of the other goes backward. Similarly, the expansion of the universe does not mean the cosmic time goes forward, and the contraction does not mean the cosmic time goes backward. The expansion or contraction is just a dynamical effect of the space-time.

2) The principles of relativity and covariance are the constraints to physical laws, but not constraints to concrete solutions. The specific matter system is only a special solution of physical laws, so the equations must be covariant, but the solutions are not definitely symmetric.

3) Time is a global property of the universe, which is a measurement of the evolution process of the universe. The universe has a unique and unified cosmic time, and it always goes unidirectionally forward. Related to this cosmic time we have the specific state of universe at some time, which forms the cosmic space and the existences. There is an internal time to reflect the evolution process of a system, just like the history and lifetime of a man, which is the proper time $\tau$ of the system. However, this proper time also takes the cosmic time as a unified background and a measuring standard.

4) The universe has a unified cosmic time $T$ and a cosmic space related to this cosmic time. The cosmic space is the total space described by the coordinate 
system relatively at rest to the cosmic background radiation. The cosmic space and time are objective and absolute. Since the total universe is also a realistic solution of all physical laws. So this solution is objective, and it does not definitely have the symmetry of the equations.

5) The eigensolution of spinor is a critical state losing time concept, which responses the interaction of environment with some uncertainty, then the evolution process of the world is not uniquely determined and has some randomness. The evolution of the world is not uniquely determined.

6) The non-uniqueness of the evolution process means that the inverse process is absent. So for a world including spinors, the evolution is essentially irreversible.

These opinions have been discussed in other papers [10-12]. In what follows, we give some detailed discussion for the structure of the space-time and relative symmetries of the transformation through specific calculations and explanations.

\section{The Quaternion Structure of Space-Time}

Relativity is a theory of space-time of the universe, which is based on the principle of constant vacuum light speed and the principles of relativity and generalized covariance. Though this treatment is reasonable is physics, there is an underlying risk in epistemology. Because the structure of space-time is a problem involving much broader contents than the vacuum light speed [13], and we can't accurately measure light speed in true vacuum. Using the property of a specific matter system to describe general concepts, it is easy to fall into the trap of language. The logical procedure should be to establish the geometry of space-time at first, and then to use the geometry and electrodynamics explaining the invariance of the vacuum light speed. In this way, a lot of plausible paradoxes will disappear, and one will not be puzzled by superluminal neutrino phenomena and problems of the time's arrow [11,12]. The theory of space-time is a geometry, so like the Euclidian geometry, it will be more effective and convenient to describe the structure of space-time by the method of geometry, and then explain the corresponding relations between realistic world and geometric concepts. In this way, the problems become simple and clear in logic. In a sense, the principle of constant light speed is a constraint for the metric of the space-time, and the principles of relativity and covariance explain the rules of transformation between coordinate systems.

A point or event in the space-time can be labeled by 3 independent spatial coordinates $x^{k}=(x, y, z)$ and one temporal coordinate $x^{0}=t$, that is to say the space-time is $3+1$ dimensional. This is a basic fact, which can only be accepted as a fundamental postulate. The selection of coordinate system and the local reference frame or tetrad at each event is subjective and arbitrary. However, the event and the 4 dimensional distance or interval between two events are objective. For the same physical system, different researchers can choose quite different coordinate system and local tetrad, but their coordinates and physical parameters should be in one-to-one correspondence, and the equations should take the same structure. This is the essential meaning of the principles of relativity and covariance. However, the usual understanding and explanations in textbooks have some problems.

Spinor equation is the most basic equation to describe matter. Detailed analysis shows that, to this kind of equation, the relativity principle is valid only if the space-time has $3+1$ dimensions and has a quadratic interval $d s^{2}$. In this case, the space-time has elegant quaternion structure, and the physical fields and their dynamic equations can be expressed in quaternion form. Their transformation laws can be realized only for such quaternion differential operator. This feature can be found in the following calculations. Further more, for this first order differential field equation system, the solutions evolve in one direction in time.

In this paper, we use notations $(a, b \in\{0,1,2,3\})$

stands for the indexes in flat space-time and $(\mu, v \in\{0,1,2,3\})$ stands for the indexes in the curved space-time, but $(j, k, l \in\{1,2,3\})$ for the indexes in space. Let $x^{\mu}=(t, x, y, z)$ be a smooth enough coordinate system labeled in the space-time and

$X^{a}\left(x^{\mu}\right)=(T, X, Y, Z)$ is an orthogonal reference system in the tangent space-time at given event $x^{\mu}$. This tetrad can be represented by the basis of Clifford algebra $C \ell_{1,3}$ which is isomorphic to the quaternion basis. The usual Dirac matrix is a realization. Denote

$$
\mathrm{d} \mathbf{X}=\gamma_{a} \mathrm{~d} X^{a}, \mathrm{~d} \mathbf{x}=\tilde{\gamma}_{\mu} \mathrm{d} x^{\mu},
$$

in which coordinate increments $\mathrm{d} x^{\mu}$ and $\mathrm{d} X^{a}$ are vectors satisfying linear transformation law. $\mathrm{d} x^{0}=\mathrm{d} t$ and $\mathrm{d} X^{0}=\mathrm{d} T$ are time-like vectors. $\tilde{\gamma}_{\mu}$ is a set of quaternion basis in curvilinear coordinate system. They are not definitely orthogonal, but satisfy the following relation

$$
\gamma^{a} \gamma^{b}+\gamma^{b} \gamma^{a}=2 \eta^{a b}, \quad \tilde{\gamma}^{\mu} \tilde{\gamma}^{v}+\tilde{\gamma}^{\mu} \tilde{\gamma}^{v}=2 g^{\mu v},
$$

where $\eta_{a b}=\operatorname{diag}[1,-1,-1,-1]$ is Minkowski metric, and $g_{\mu \nu}$ is the metric in curvilinear coordinate system,

$$
\begin{cases}\tilde{\gamma}^{\mu}=h_{a}^{\mu} \gamma^{a}, & \tilde{\gamma}_{\mu}=l_{\mu}^{a} \gamma_{a}, \quad h_{a}^{\mu} l_{\mu}^{b}=h_{k}^{b} l_{a}^{k}=\delta_{a}^{b}, \\ \mathrm{~d} x^{\mu}=h_{a}^{\mu} \mathrm{d} X^{a}, & \partial_{x^{\mu}}=l_{\mu}^{a} \partial_{X}, \\ g^{\mu \nu}=h_{a}^{\mu} h_{b}^{v} \eta^{a b}, & g_{\mu v}=l_{\mu}^{a} l_{v}^{b} \eta_{a b} .\end{cases}
$$


The calculation and explanation for the tetrad coefficients $\left(h_{a}^{\mu}, l_{\mu}^{a}\right)$ refer to $[14,15]$.

The interval between two infinitely adjacent events $\mathrm{d} \mathbf{x}^{2}$ or $\mathrm{dX}^{2}$ is an objective quantity independent of coordinate system, we have

$$
\begin{aligned}
\mathrm{d} s^{2} & =\mathrm{d} \mathbf{x}^{2}=\frac{1}{2}\left(\tilde{\gamma}_{\mu} \tilde{\gamma}_{v}+\tilde{\gamma}_{v} \tilde{\gamma}_{\mu}\right) \mathrm{d} x^{\mu} \mathrm{d} x^{v}=g_{\mu \nu} \mathrm{d} x^{\mu} \mathrm{d} x^{v} \\
& =\mathrm{d} \mathbf{X}^{2}=\frac{1}{2}\left(\gamma_{a} \gamma_{b}+\gamma_{b} \gamma_{a}\right) \mathrm{d} X^{a} \mathrm{~d} X^{b}=\eta_{a b} \mathrm{~d} X^{a} \mathrm{~d} X^{b} .
\end{aligned}
$$

(2.1) and (2.4) are the fundamental postulates on the space-time, and all results of the special relativity can be derived from them [12].

The properties of a fundamental physical system can be described by fields and interactive coefficients. For example, a particle can be described by spinor $\phi$, electromagnetic field by vector potential $A^{\mu}$ and field intensity $(\boldsymbol{E}, \boldsymbol{B})$, and gravitational field by metric $g_{\mu v}$. For a relatively isolated system, we can express all fields to describe the system by a column vector $\Psi=\left(\psi_{1}, \psi_{2}, \cdots, \psi_{n}\right)^{T}$, then the dynamical equation of the system certainly take the following form [16],

$$
\partial \Psi=f(\Psi), \quad \partial \equiv \tilde{\gamma}^{\mu} \partial_{\mu},
$$

in which $\partial$ is a quaternion differential operator, $f(\Psi)$ consists of some tensorial products of $\Psi$, such that the total equation satisfies covariance. The following calculations show that, the principles of relativity and covariance completely depend on the perfect and marvellous quaternion structure of (2.5). The covariance is invalid in other dimensional space-time.

The quaternion basis $\tilde{\gamma}^{\mu}$ in operator $\partial=\tilde{\gamma}^{\mu} \partial_{\mu}$ can be represented by matrices. The minimum order is 4 , and the usual Dirac matrices are a specific realization. It can be proved that the order of the matrices is an integral multiple of 4, so the dimension $n$ of field

$\Psi=\left(\psi_{1}, \psi_{2}, \cdots, \psi_{n}\right)^{T}$ is also the integral multiple of 4 . In fact, all physical phenomena we have observed can be described by spinor $\phi$ with 4 components and spin

$s=\frac{1}{2}$, vector $(A, \tilde{A})$ with 8 components and spin $s=1$, and tensor $\left(G=\left(g_{\mu \nu}\right), \tilde{G}\right)$ with 32 components and spin $s=2$ together with their combinations. These fields all exist in couples form. Between different representations, the operator $\partial$ and $\Psi$ should make a double linear transformation. This transformation reflects the different selection of coordinates and measuring methods of fields.

Denote the Pauli matrices by

$$
\begin{gathered}
\sigma^{a} \equiv\left\{\left(\begin{array}{ll}
1 & 0 \\
0 & 1
\end{array}\right),\left(\begin{array}{ll}
0 & 1 \\
1 & 0
\end{array}\right),\left(\begin{array}{cc}
0 & -i \\
i & 0
\end{array}\right),\left(\begin{array}{cc}
1 & 0 \\
0 & -1
\end{array}\right)\right\}, \\
\tilde{\sigma}^{a} \equiv\left(\sigma^{0},-\sigma\right), \quad \sigma=\left(\sigma^{1}, \sigma^{2}, \sigma^{3}\right) .
\end{gathered}
$$

then the base vector $\gamma^{a}$ of 4 order can be represented by the following matrices with symmetry,

$$
\gamma^{a} \equiv\left(\begin{array}{cc}
0 & \sigma^{a} \\
\tilde{\sigma}^{a} & 0
\end{array}\right)=\left\{\left(\begin{array}{cc}
0 & I \\
I & 0
\end{array}\right),\left(\begin{array}{cc}
0 & \boldsymbol{\sigma} \\
-\boldsymbol{\sigma} & 0
\end{array}\right)\right\} .
$$

This representation is equivalent to the usual Dirac matrix. But in contrast with Dirac matrix, (2.8) can display the symmetry of the fields. The coefficient matrix of (2.5) can be constructed by the block matrix of (2.8).

Dynamical Equation (2.5) is a first order symmetric hyperbolic evolution equation system. Its general solution can be expressed by the following integral,

$$
\Psi\left(t, x^{k}\right)=\int_{t_{0}}^{t} \mathrm{~d} \tau \int_{\Omega\left(\tau, x^{k}\right)}\left(F+K^{j} \partial_{j} \Psi\left(\tau, \xi^{k}\right)\right) \mathrm{d} \xi,
$$

where $t_{0}$ is any given time smaller than the present time $t, t_{0}<t, \cup_{\tau \in\left[t_{0}, t\right)} \Omega\left(\tau, x^{k}\right)$ is the dependent domain of $\Psi\left(t, x^{k}\right)$, which is included in the light cone with vertex $\left(t, x^{k}\right)$, and

$$
F=F\left(\Psi\left(\tau, \xi^{k}\right)\right), \quad K^{j}=K^{j}\left(\Psi\left(\tau, \xi^{k}\right)\right) .
$$

The representation (2.9) is solved by characteristic cone method. The geometrical meaning is shown in Figure 1. The representation (2.9) manifestly reveals some philosophical features such as the arrow of time, causal and historical connection. This is the reason why to choose the formula as a fundamental physical postulate to replace (2.5) [16].

In Figure 1, the cosmic space is absolute space of the universe, which is described by the coordinate system relatively rest to the cosmic background radiation. Cosmic time is the absolute time. The absolute space is a simultaneous hyperplane relative to the cosmic time. The cosmic time has unitarity and uniqueness. $P$ stands for a particle moving in the cosmic space, but $A, B, O, Q$ stand for points relatively rest to the cosmic space. The coordinates $x^{\mu}=(t, x, y, z)$ is not definitely orthogonal, but we can establish orthogonal local tetrad

$\mathrm{d} X^{a}=(\mathrm{d} T, \mathrm{~d} X, \mathrm{~d} Y, \mathrm{~d} Z)$ at each event $x^{\mu}$, and (3) gives the relation between $\mathrm{d} X^{a}$ and $\mathrm{d} x^{\mu} . \Omega\left(t, t_{0}, Q\right)$ stands for dependent domain of point $Q$ from time $t_{0}$ to $t$, which means the state and physical parameters at point $Q$ is determined by the historical data in this domain. $\Omega\left(t, t_{0}, A-B\right)$ is the dependent domain of region $A-B$ from $t_{0}$ to $t$. Due to the local correlation of interaction, $t_{0}$ can be any given time smaller than present time $t$, such as $t_{1}$ or $t_{2}$.

From the space-time diagram, we can get the following conclusions:

1) The unidirectionality of time: The general solution (2.9) reflects the causal connections between parameters in field Equation (2.5). It evidently shows the unidirectionality and sequentiality of time. The future is always 


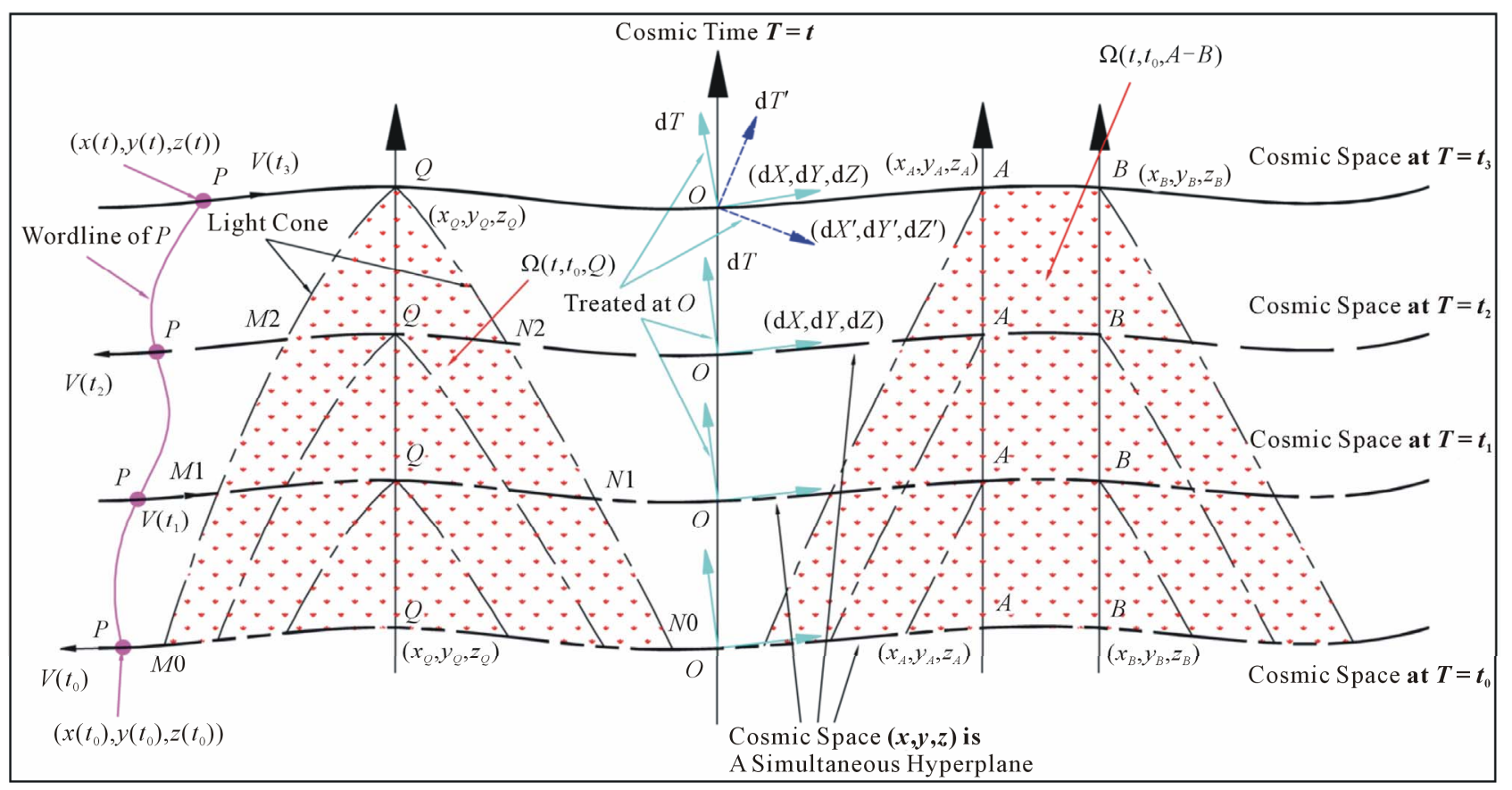

Figure 1. Space-time diagram: The cosmic space is the absolute space of the universe, which is describe by the coordinate system relatively at rest to the cosmic background radiation. Cosmic time is the absolute time. The absolute space is a simultaneous hyperplane relative to the cosmic time. The cosmic time has unitarity and uniqueness. $P$ stands for a mass point moving in the cosmic space, but $A, B, O, Q$ stand for points relatively at rest to the cosmic space. The coordinates $x^{\mu}=(t, x, y, z)$ is not definitely orthogonal, but we can establish orthogonal local tetrad $\mathrm{d} X^{a}=(\mathrm{d} T, \mathrm{~d} X, \mathrm{~d} Y, \mathrm{~d} Z)$ at each event $x^{\mu}$, and (3) gives the relation between $\mathrm{d} X^{a}$ and $\mathrm{d} x^{\mu} . \Omega\left(t, t_{0}, Q\right)$ stands for dependent domain of point $Q$ from time $t_{0}$ to $t$, and $\Omega\left(t, t_{0}, A-B\right)$ for dependent domain of region $A-B$ from $t_{0}$ to $t$.

determined by the past. The fields of $Q$ at time $t$ are only determined by the history in the backward characteristic cone. Future and past can not be mixed. The particle $P$ moves in left direction at $t_{0}$ and $t_{2}$, and in right direction at $t_{1}$ and $t$. The motion can be reversed, but the cosmic time $\mathcal{T}$ goes always unidirectionally forward. The inversion of process just means the reflection of motion, rather than inversion of time. The arrow of time has nothing to do with the expansion or contraction of the universe, which is just a dynamical effect of the space-time. The unidirectionality of time is an objective property of the universe.

Since the eigen state of a spinor is a critical state without unique evolutionary direction, the non-uniqueness of the process makes the inverse of evolution become impossible. A thermodynamics system consists of a tremendous number of microscopic particles all described by spinors. And the evolution of each particle has some uncertainty or randomness, so the thermodynamic process is essentially irreversible. Entropy can be only clearly defined for a system satisfying large number statistical assumption. But for a system with internal structure, the entropy can not be defined. It is the randomness of a statistical system which defines the entropy, rather than the entropy decides the irreversible process [11].

2) The absoluteness of space-time: By Figure 1 we learn, the evolution of the universe has globality and synchronism. There exists a global cosmic time scale $\mathcal{T}$, whose meaning is close to the concept of Newtonian absolute time. When $\mathcal{T}$ evolves to some specific moment $t_{0}$, it corresponds to the fact that universe evolves to a specific state. This specific state is a simultaneous hyperplane which defines the cosmic space. The meaning of the cosmic space is close to the concept of Newtonian absolute space. The space-time itself and the matter inside it all evolve from one hyperplane to next hyperplane. The cosmic time can neither go back, nor be transcended.

3) The subjectivity of choice coordinate and tetrad system: Does the absoluteness of the space-time contradicts relativity? The answer is NO. Different from the usually understanding that the relativity denied the objectivity of time and space, the significance of relativity theory is that it emphasizes the correlation between space and time, and the universality of the principle of relativity and covariance. To understand this, we must firstly understand "The realistic world is just a solution 
of physical laws, and this solution is objective existence". The laws of physics can also have many other reasonable and realizable solutions, but they are not objective existence. What the covariance emphasizes is that, the coordinate system is just an identification system which can be quite arbitrary chosen. Between different coordinate systems there is 1-1 corresponding transformation. Only in the coordinate system relatively rest to the cosmic background radiation we have objective simultaneous hyperplane and realistic global simultaneity. In the local frame and curvilinear coordinate system moving relative to the cosmic background radiation, the simultaneous hyperplane is only of theoretical sense, rather than objective existence.

4) The uniqueness of simultaneity: This problem has been explained in $[11,12]$, which can be illustrated by Figure 2.

Assume that we have realistic simultaneous hyperplane in the static reference frame $S(T, X, Y, Z)$, and at moment $T=0$, we have simultaneous hyperplane (i.e. the space of the world) $A^{\prime} B^{\prime}$, which evolves into $C^{\prime} D^{\prime}$ at moment $T=T_{1}$ and $E^{\prime} F^{\prime}, \cdots$. By Lorentz transformation, this real space corresponds to the inclined plane $A B, C D, E F, \cdots$ respectively in $O(t, x, y, z)$. The simultaneous hyperplane in $O(t, x, y, z)$ is the dash lines. The dash lines can not be realistic simultaneous hyperplane, because the part with $x<0$ has become history in view of $S(T, X, Y, Z)$, but not yet been determined in view of $O(t, x, y, z)$ and could be designed and chosen. On the other hand, the part $x>0$ in $S(T, X, Y, Z)$ does not take place yet, but it has become history in $O(t, x, y, z)$. The situation is absurd, because the evolution of the universe is not uniquely determined. The simultaneous hyperplane in $S(T, X, Y, Z)$ and $O(t, x, y, z)$ describe two different

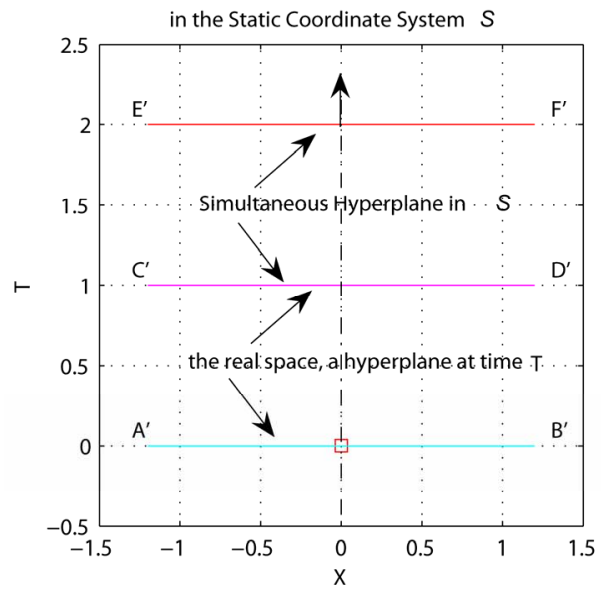

worlds. In this sense, the proposition "all inertial frames of reference are equivalent" is invalid.

5) The internal time of matter system: To understand time, we should distinguish the different meanings of cosmic time $\mathcal{T}$ and the internal time or proper time $\tau$ of a specific matter system. $\mathcal{T}$ is a measurement for the evolution process of the whole universe, and it is objective and acts as a global standard of time. But $\tau$ is a measurement of the evolution speed of a specific matter system. Relativity provides the method to treat the relationship between $\mathcal{T}$ and $\tau$, that is,

$$
\Delta \tau=\int_{T_{0}}^{T} \sqrt{1-v^{2}} \mathrm{~d} \mathcal{T} .
$$

\section{Transformation Law and the Physical Meaning}

Dynamical Equation (2.5) simultaneously has general covariance under curvilinear coordinate transformation and Lorentz invariance under transformation of local frame $\gamma^{a}$. For vectors and tensors, since they can be directly projected to the natural basis of the coordinate system $\partial_{\mu}$, the representation is simple. It is enough that the equation takes tensorial form. However, for the spinors the problem becomes complicated and abstruse, because the spinor is related to concrete local tetrad. In what follows, we examine the physical meaning of the transformation and the quaternion structure of spinor and space-time via specific calculation.

\subsection{Dirac Equation in Spherical Coordinate System}

In [15], we derived the covariant form of the spinor equation in spherical coordinate system,

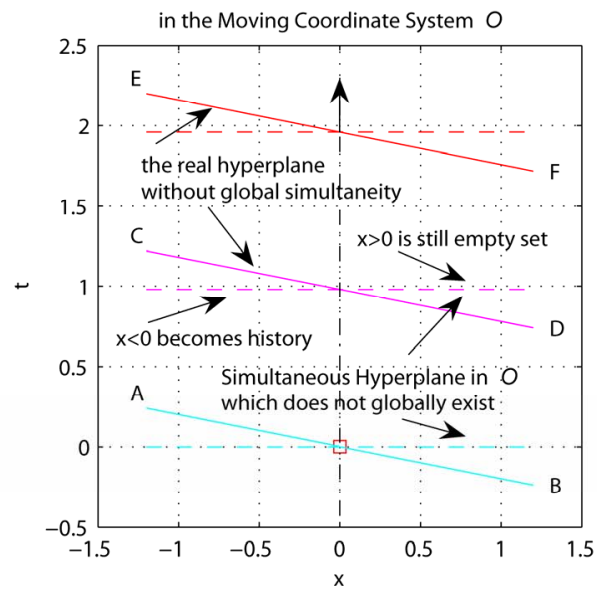

Figure 2. The real space is an evolution simultaneous hyperplane: In the static reference frame $S$, the simultaneous hyperplane is $A^{\prime} B^{\prime}, C^{\prime} D^{\prime}, E^{\prime} F^{\prime}$ etc. But in moving reference frame $O$, it corresponds to inclined hyperplane $A B, C D, E F$. The simultaneous hyperplane in reference frame $O$ is the dash lines. The simultaneous hyperplanes in two reference frame can not be all realistic for a world. 


$$
\begin{aligned}
& i\left(\gamma^{0} \partial_{t}+\gamma^{1}\left(\partial_{r}+\frac{1}{r}\right)+\frac{\gamma^{2}}{r}\left(\partial_{\theta}+\frac{1}{2} \cot \theta\right)+\frac{\gamma^{3}}{r \sin \theta} \partial_{\varphi}\right) \phi \\
& =m \phi .
\end{aligned}
$$

But some readers told that it is different from the equation directly transformed from the Cartesian Coordinate system. They felt that it is not understandable and even doubted whether there is error. Now we derive the covariant equation by direct calculation, and show the meaning of tetrad basis $\gamma^{a}$. We represent the bispinor $\phi$ in the Weyl form $\psi$ and $\tilde{\psi}$. This is the so called chiral form. Then the Dirac equation in Cartesian coordinate system becomes,

$$
\left\{\begin{array}{l}
i\left(\partial_{t}+\boldsymbol{\sigma} \cdot \nabla\right) \psi=m \tilde{\psi} \\
i\left(\partial_{t}-\sigma \cdot \nabla\right) \tilde{\psi}=m \psi
\end{array}\right.
$$

in which $\nabla=\left(\partial_{x}, \partial_{y}, \partial_{z}\right)$. Transform the Cartesian coordinates $(x, y, z)$ into spherical coordinates $(r, \theta, \varphi)$, by straightforward calculation we have

$$
\boldsymbol{\sigma} \cdot \nabla=\sigma_{r} \partial_{r}+\frac{1}{r} \sigma_{\theta} \partial_{\theta}+\frac{1}{r \sin \theta} \sigma_{\varphi} \partial_{\varphi},
$$

where $\left(\sigma_{r}, \sigma_{\theta}, \sigma_{\varphi}\right)$ reads

$$
\left\{\begin{array}{l}
\left(\begin{array}{cc}
\cos \theta & \sin \theta e^{-\varphi i} \\
\sin \theta e^{\varphi i} & -\cos \theta
\end{array}\right), \\
\left.\left(\begin{array}{cc}
-\sin \theta & \cos \theta e^{-\varphi i} \\
\cos \theta e^{\varphi i} & \sin \theta
\end{array}\right),\left(\begin{array}{cc}
0 & -i e^{-\varphi i} \\
i e^{\varphi i} & 0
\end{array}\right)\right\} .
\end{array}\right.
$$

Then the dynamical Equation (3.2) becomes

$$
\left\{\begin{array}{l}
i\left(\partial_{t}+\sigma_{r} \partial_{r}+\frac{1}{r} \sigma_{\theta} \partial_{\theta}+\frac{1}{r \sin \theta} \sigma_{\varphi} \partial_{\varphi}\right) \psi=m \tilde{\psi}, \\
i\left(\partial_{t}-\sigma_{r} \partial_{r}-\frac{1}{r} \sigma_{\theta} \partial_{\theta}-\frac{1}{r \sin \theta} \sigma_{\varphi} \partial_{\varphi}\right) \tilde{\psi}=m \psi .
\end{array}\right.
$$

$$
\begin{array}{rr}
\Pi=\Pi^{*}=\frac{1}{\sqrt{2}}\left(\begin{array}{rr}
i \exp \left[-\frac{i}{2}\left(\theta+\varphi+\frac{\pi}{2}\right)\right], & \exp \left[\frac{i}{2}\left(\theta-\varphi+\frac{\pi}{2}\right)\right] \\
-\exp \left[-\frac{i}{2}\left(\theta-\varphi+\frac{\pi}{2}\right)\right], & -i \exp \left[\frac{i}{2}\left(\theta+\varphi+\frac{\pi}{2}\right)\right]
\end{array}\right) \\
\Pi^{-1}=\frac{1}{\sqrt{2}}\left(\begin{array}{rr}
-i \exp \left[\frac{i}{2}\left(\theta+\varphi+\frac{\pi}{2}\right)\right], & -\exp \left[\frac{i}{2}\left(\theta-\varphi+\frac{\pi}{2}\right)\right] \\
\exp \left[-\frac{i}{2}\left(\theta-\varphi+\frac{\pi}{2}\right)\right], & i \exp \left[-\frac{i}{2}\left(\theta+\varphi+\frac{\pi}{2}\right)\right]
\end{array}\right)
\end{array}
$$

Substituting (3.7)-(3.11) into original Equation (3.5), we can get the following form by some arrangement.

$$
\left\{\begin{array}{l}
i\left[\sigma^{0} \partial_{t}+\sigma^{1}\left(\partial_{r}+\frac{1}{r}\right)+\frac{1}{r} \sigma^{2}\left(\partial_{\theta}+\frac{1}{2} \cot \theta\right)+\frac{1}{r \sin \theta} \sigma^{3} \partial_{\varphi}\right] \psi^{\prime}=m \tilde{\psi}^{\prime}, \\
i\left[\sigma^{0} \partial_{t}-\sigma^{1}\left(\partial_{r}+\frac{1}{r}\right)-\frac{1}{r} \sigma^{2}\left(\partial_{\theta}+\frac{1}{2} \cot \theta\right)-\frac{1}{r \sin \theta} \sigma^{3} \partial_{\varphi}\right] \tilde{\psi}^{\prime}=m \psi^{\prime} .
\end{array}\right.
$$


(3.12) is just the same equation calculated in [15]. The above calculation and equation show that,

1) The tetrad coefficient matrix is given by

$$
\begin{aligned}
& \left(h_{a}^{\mu}\right)=\operatorname{diag}\left(1,1, \frac{1}{r}, \frac{1}{r \sin \theta}\right), \\
& \left(l_{\mu}^{a}\right)=\operatorname{diag}(1,1, r, r \sin \theta) .
\end{aligned}
$$

The following vectors are orthogonal,

$$
\begin{aligned}
\partial_{X^{a}} & =\left(\partial_{t}, \partial_{r}, \frac{1}{r} \partial_{\theta}, \frac{1}{r \sin \theta} \partial_{\varphi}\right), \\
\mathrm{d} X^{a} & =(\mathrm{d} t, \mathrm{~d} r, r \mathrm{~d} \theta, r \sin \theta \mathrm{d} \varphi) .
\end{aligned}
$$

2) By spinor transformation (3.7), the direction of the tetrad basis turns into: $\gamma^{0}$ along $\mathrm{d} t, \gamma^{1}$ along $\mathrm{d} r$, $\gamma^{2}$ along $\mathrm{d} \theta$, and $\gamma^{3}$ along $\mathrm{d} \varphi$ direction, because in the tangent space

$$
\mathrm{d} X^{a} \equiv(\mathrm{d} T, \mathrm{~d} X, \mathrm{~d} Y, \mathrm{~d} Z)=(\mathrm{d} t, \mathrm{~d} r, r \mathrm{~d} \theta, r \sin \theta \mathrm{d} \varphi),
$$

and (3.12) becomes

$$
\left\{\begin{array}{l}
i\left(\sigma^{0} \partial_{T}+\sigma^{1} \partial_{X}+\sigma^{2} \partial_{Y}+\sigma^{3} \partial_{Z}+\Gamma\right) \psi^{\prime}=m \tilde{\psi}^{\prime}, \\
i\left(\sigma^{0} \partial_{T}-\sigma^{1} \partial_{X}-\sigma^{2} \partial_{Y}-\sigma^{3} \partial_{Z}+\tilde{\Gamma}\right) \tilde{\psi}^{\prime}=m \psi^{\prime} .
\end{array}\right.
$$

3) The transformation law between displacement vector $\mathrm{d} x=\gamma_{a} \mathrm{~d} x^{a}$ and $\mathrm{d} X=\gamma_{a} \mathrm{~d} X^{a}$, and between the differential operators $\partial_{X}=\gamma^{a} \partial_{x^{a}}$ and $\partial_{X}=\gamma^{a} \partial_{X^{a}}$ is given by [12],

$$
\mathrm{d} x=\gamma^{0} \Omega \mathrm{d} X \Omega \gamma^{0}, \quad \partial_{x}=\gamma^{0} \Omega \partial_{X} \Omega \gamma^{0},
$$

where $\Omega=w_{a} \gamma^{a}$, and the operator $\partial_{X}$ does not make derivatives on $\Omega$.

\subsection{Transformation Law under the Reflection of Tetrad}

Under curvilinear coordinate transformation $x^{\mu} \rightarrow x^{\prime \mu}$, the transformation of tetrad $\tilde{\gamma}^{\mu}=h_{a}^{\mu} \gamma^{a}$ is realized via the index $\mu$ of coefficient matrix $h_{a}^{\mu}$, but the spinor does not transform in form. However, the definition of covariant derivative of spinor actually implies a transformation like (3.7). Under proper Lorentz transformation of tetrad, the transformation of spinor is similar to (3.7), and can be easily derived. In what follows, we check the invariance and transformation laws of the spinor equation with interactions under the tetrad reflection transformation.

Denote $4 \times 4$ Hermitian matrices by

$$
\alpha^{\mu}=\left\{\left(\begin{array}{ll}
I & 0 \\
0 & I
\end{array}\right),\left(\begin{array}{ll}
0 & \sigma \\
\sigma & 0
\end{array}\right)\right\}, \beta=\left(\begin{array}{cc}
0 & I \\
I & 0
\end{array}\right), \gamma=\left(\begin{array}{cc}
I & 0 \\
0 & -I
\end{array}\right) \text {, }
$$

we examine the following Lagrangian

$$
L=\phi^{+} \alpha^{\mu}\left(i \partial_{\mu}-e A_{\mu}\right) \phi-\mu c \breve{\gamma}+\frac{1}{2} w \breve{\gamma}^{2}-\frac{1}{2} \partial_{\mu} A_{\nu} \partial^{\mu} A^{v},
$$

in which $A^{\mu}$ is the electromagnetic potential,

$$
\breve{\alpha}^{\mu}=\phi^{+} \alpha^{\mu} \phi, \quad \breve{\gamma}=\phi^{+} \gamma \phi .
$$

According to the transformation of $\phi$, it is easy to prove that, $\breve{\alpha}^{\mu}$ is a contravariant vector, and $\bar{\gamma}$ a true scalar. The corresponding dynamical equation is given by,

$$
\begin{aligned}
\alpha^{\mu}\left(\hbar i \partial_{\mu}-e A_{\mu}\right) \phi & =(\mu c-w \breve{\gamma}) \gamma \phi, \\
\partial_{\alpha} \partial^{\alpha} A^{\mu} & =e q^{\mu},
\end{aligned}
$$

in which $q^{\mu} \equiv \breve{\alpha}^{\mu}$ is the current of spinor. The Hamiltonian form of (3.20) is given by,

$$
\hbar i \partial_{t} \phi=\hat{H} \phi, \quad \hat{H}=c\left[e A_{0}+\boldsymbol{\alpha} \cdot \hat{p}+(\mu c-w \breve{\gamma}) \gamma\right] .
$$

where $\hat{p}=-\hbar i \nabla+e \boldsymbol{A}$ is the momentum operator. For (3.22) we have normalizing condition,

$$
\int_{R^{3}}|\phi|^{2} \mathrm{~d}^{3} x=1 .
$$

Now we check the invariance of (3.22) under space reflection transformation. Making double linear transformation

$$
t=t^{\prime}, \quad \boldsymbol{x}=-\boldsymbol{x}^{\prime}, \quad \phi(t, \boldsymbol{x})=\gamma \phi^{\prime}\left(t^{\prime}, \boldsymbol{x}^{\prime}\right),
$$

where an indeterminate gauge factor $e^{\xi i}$ is ignored. Easy to check $\boldsymbol{\alpha} \gamma=-\gamma \boldsymbol{\alpha},(\gamma)^{2}=I, \nabla=-\nabla^{\prime}$ and $\hat{p}=-\hat{p}^{\prime}$,

$$
\begin{aligned}
& q^{\mu} \equiv \phi^{+} \alpha^{\mu} \phi=\phi^{\prime+} \gamma \alpha^{\mu} \gamma \phi^{\prime}=\left(q^{\prime 0},-q^{\prime 1},-q^{\prime 2},-q^{\prime 3}\right), \\
& \breve{\gamma}^{\prime}=\phi^{+}(\gamma)^{3} \phi=\widetilde{\gamma} .
\end{aligned}
$$

That is $q^{\prime 0}=q^{0}$ and $q^{\prime k}=-q^{k},(k=1,2,3)$. Substituting (3.25) into (3.21) we get the transformation of $A^{\mu}$,

$$
A^{\prime 0}=A^{0}, \quad \boldsymbol{A}^{\prime}=-\boldsymbol{A} .
$$

Of course, (3.25) and (3.26) are just the transformation of a contravariant vector under space reflection.

Substituting the above relations into (3.22), we get

$$
\begin{aligned}
\hbar i \partial_{t^{\prime}} \phi^{\prime} & =c \gamma\left[e A_{0}^{\prime}-\boldsymbol{\alpha} \cdot \hat{p}^{\prime}+(\mu c-w \breve{\gamma}) \gamma\right] \gamma \phi^{\prime}, \\
& =c\left[e A_{0}^{\prime}+\boldsymbol{\alpha} \cdot \hat{p}^{\prime}+\left(\mu c-w \bar{\gamma}^{\prime}\right) \gamma\right] \phi^{\prime} .
\end{aligned}
$$

The Equation (3.27) takes the same form of (3.22). This means the law for this spinor is independent of reference frame $\left(t^{\prime}, \boldsymbol{x}^{\prime}\right)$ or $(t, \boldsymbol{x})$, and the transformation for field satisfies invertible linear relation (3.24). This is true meaning of principle of relativity.

Now we check the invariance under time inversion transformation. Making double linear transformation, 


$$
t=-t^{\prime}, \quad \boldsymbol{x}=\boldsymbol{x}^{\prime}, \quad \phi(t, \boldsymbol{x})=\alpha_{2} \beta \overline{\phi^{\prime}}\left(t^{\prime}, \boldsymbol{x}^{\prime}\right) .
$$

Easy to prove

$$
\nabla=\nabla^{\prime}, \beta \alpha^{\mu}=\alpha^{\mu} \beta, \beta \gamma=-\gamma \beta, \beta \alpha_{2} \gamma=\gamma \alpha_{2} \beta
$$

and

$$
\begin{aligned}
& \beta \alpha_{2}\left(\alpha^{1}, \alpha^{2}, \alpha^{3}\right)=\left(-\alpha^{1}, \alpha^{2},-\alpha^{3}\right) \alpha_{2} \beta, \\
& \bar{\gamma}^{\prime}=\bar{\phi}^{+} \beta \alpha_{2} \gamma \alpha_{2} \beta \bar{\phi}=\breve{\gamma} . \\
q^{\mu} \equiv & \phi^{+} \alpha^{\mu} \phi=\bar{\phi}^{\prime+} \beta \alpha_{2} \alpha^{\mu} \alpha_{2} \beta \bar{\phi}^{\prime} \\
= & \bar{\phi}^{\prime+} \alpha_{2} \alpha^{\mu} \alpha_{2} \bar{\phi}^{\prime}=\bar{\phi}^{\prime+}\left(\alpha^{0},-\alpha^{1}, \alpha^{2},-\alpha^{3}\right) \bar{\phi}^{\prime} \\
= & \overline{\phi^{\prime+}\left(\alpha^{0},-\alpha^{1},-\alpha^{2},-\alpha^{3}\right) \phi^{\prime}} \\
= & \left(q^{\prime 0},-q^{\prime 1},-q^{\prime 2},-q^{\prime 3}\right) .
\end{aligned}
$$

From (3.30) we learn, the transformation law of contravariant vector $q^{\mu}$ under time inversion is different from that under curvilinear coordinate transformation $q^{\prime 0}=-q^{0}, \boldsymbol{q}^{\prime}=\boldsymbol{q}$. It takes the same form under space reflection transformation.

Substituting (3.30) into (3.21) we get the transformation law of potential $A^{\prime 0}=A^{0}, \boldsymbol{A}^{\prime}=-\boldsymbol{A}$. For operator $\hat{p}$ we have

$$
\hat{p}=-\hbar i \nabla+e A=-\hbar i \nabla^{\prime}-e A^{\prime}=-\overline{\hat{p}}^{\prime} .
$$

Substituting the above relations into (3.22), we get

$$
\begin{aligned}
& -\hbar i \partial_{t^{\prime}} \bar{\phi}^{\prime} \\
& =c \beta \alpha_{2}\left[e A_{0}+\alpha \cdot \hat{p}^{\prime}+(\mu c-w \breve{\gamma}) \gamma\right] \alpha_{2} \beta \overline{\phi^{\prime}}, \\
& =c\left[e A_{0}^{\prime}+\left(-\alpha^{1}, \alpha^{2},-\alpha^{3}\right) \cdot\left(-\overline{\hat{p}^{\prime}}\right)+\left(\mu c-w \bar{\gamma}^{\prime}\right) \gamma\right] \overline{\phi^{\prime}} .
\end{aligned}
$$

Take the complex conjugate of (3.32), we get

$$
\hbar i \partial_{t^{\prime}} \phi^{\prime}=c\left[e A_{0}^{\prime}+\vec{\alpha} \cdot \hat{p}^{\prime}+\left(\mu c-w \breve{\gamma}^{\prime}\right) \gamma\right] \phi^{\prime} .
$$

(3.33) also takes the same form of (3.22), so the spinor and vector equations all keep the invariance under the space-time inversion transformation.

From the above calculations we learn,

1) For the first order Equation (2.5), the principle of relativity and covariance is a strong constraints, which can be realized only in $3+1$ dimensional space-time due to the quaternion structure. In this point of view, the theories with other dimensions are almost impossible in physics. The fact that the unverse chooses the $3+1$ dimensional space-time with elegant quaternion structure has profound philosophical implications, which is the sapiential design of the Creator.

2) The philosophical reason of the principle of relativity and covariance is the arbitrariness and subjectiveness to choose the coordinates and measurement method of fields. Charge is a property of a specific matter system. One particle carries a positive or a negative charge or even no charge is an objective fact, which can not be transformed. In this point of view, the so called CPT invariance theorem is simply not a physical law, but a result due to misusing concepts. For a specific spinor equation, the change of charge means the object being described also changed, and the equation is describing another system. Of course, as a mathematical theorem to show the relation between solutions and coefficients is reasonable.

3) The function of quaternion basis is similar to the unit of imaginary number $i$. They are concrete numbers, so they can not be transformed under coordinate or tetrad transformation. In quantum field theory we have transformations such as $\hat{T} i \hat{T}^{-1}=-i, \hat{\boldsymbol{T}} \boldsymbol{\alpha} \hat{T}^{-1}=-\boldsymbol{\alpha}$. These relations should be a results of misunderstanding the meaning of principle of relativity and misusing concepts. The reason why we have such transformations seems to be we need them. Nobody can really understand such illegal relations [17].

The tetrad coefficients $h_{a}^{\mu}$ or $l_{\mu}^{a}$ is different from $\gamma^{a}$. They are fields similar to metric $g_{\mu \nu}$, so $\tilde{\gamma}^{\mu}=h_{a}^{\mu} \gamma^{a}$ is transformed under curvilinear coordinate transformation. However in this case the transformation of spinors is implicit, and the possible transformation is included in the definition of spinor covariant derivative [15].

4) Chiral Equation (3.2) is equivalent to the usual Dirac equation, and the transformation just means the method of measuring field is different. Just like changing coordinates does not influence the property of space-time, the transformation of field also does not influence the property of this concrete particle. The representation $\phi$ and $(\psi, \tilde{\psi})$ only needs an invertible linear transformation,

$$
\phi=\frac{1}{\sqrt{2}}\left(\begin{array}{cc}
I & I \\
I & -I
\end{array}\right)\left(\begin{array}{l}
\psi \\
\tilde{\psi}
\end{array}\right) .
$$

Such transformation can not increase any new phenomenon. For the same equation, it is impossible that the equation describes electron if the spinor is denoted by $\phi$, and describes neutrino when $\phi$ is transformed into $(\psi, \tilde{\psi})$. Because physical phenomenon is objective, but the description is subjective. Electrons and neutrino have different internal structure, they should be described by different equations with different interacting coefficients.

\section{Some Problems Relative to the Structure of Space-Time}

\subsection{Hyperdrive Neutrino}

It was reported that the hyperdrive neutrino phenomenon was observed in supernova explosion and OPERA experiment [18]. This leads to the doubt of the effectiveness of the theory of relativity. However, the problem may be not in the foundation of relativity, but in the 
expression of the theory. From the above calculation we find that, the $3+1$ dimensional space-time has special advantages, which should be described by quaternion geometry. The traditional theory of relativity starts from the principle of constant vacuum speed of light. This isn't illegal in principle. But for the following reasons it is easy to cause difficulty in understanding: 1) The electromagnetic interaction is of "viscosity". The light always slows down when it goes through medium due to the internal dielectric constant of the medium. Since we can never really get the absolute vacuum for electromagnetic field, we can not get exact vacuum speed of light. The measured speed of light is always less than the theoretical vacuum light speed. 2) Except for the electromagnetic field, we have a lot of other fields such as spinors, gravitational field and strong interaction field in the space-time. Their propagating speed is also vacuum speed of light determined by the quaternion differential operator $\partial=\gamma^{a} \partial_{a}$. So it is more natural to establish geometry of the space-time at first, and then explain the speed of light according to geometry and electrodynamics. Otherwise, we may fall into the trap of language.

To explain the electromagnetic "viscosity" in vacuum, we must make clear the annihilation and creation of particle and anti-particle at first $[19,20]$. We know that positrons and electrons are all described by spinors, which all satisfy the normalization condition. So the spinors can never be really annihilated. The encounter of particle and anti-particle is different from the summation of their electromagnetic interaction coefficients $+e-e=0$. They are encounter of spinors, and for spinors we always have $\phi_{+e}+\phi_{-e} \neq 0$. The encounter of particle and anti-particle, like the encounter of electron and proton, will form a tight neutral core. Such neutral core only has extremely weak interaction with other particles, which forms a kind of pseudo dark matter dispersed in the universe. In appropriate conditions and interacted by high-energy photons, the neutral core will be broken again. So for electromagnetic interaction we can never get the absolute vacuum. How can we get the accurate vacuum speed of light?

\subsection{The Fine Structure of Mass-Energy Relation}

Only in Newtonian mechanics, mass is a basic concept, and the value is determined by empirical data. However, mass is not a basic concept in other fundamental theory of physics, which must be derived from other equations or relations, and the value depends on the context theory. This problem leads to a lot of misunderstanding and controversy. In the author's point of view, only fields are the basic concept for elementary particles, and other properties and relations should be derived from the dynamical equation of fields. In [20-22] we found the mass-speed relation for a spinor with interaction potential is not the simple Einstein mass-energy relation $E=m c^{2}$, which has fine structure,

$$
E=\frac{M_{0}}{\sqrt{1-v^{2}}}-\frac{M_{1} v^{2}}{\sqrt{1-v^{2}}}+\frac{W_{F}}{\sqrt{1-v^{2}}} \ln \frac{1}{\sqrt{1-v^{2}}},
$$

where $M_{0}, M_{1}, W_{F}$ are positive constants with mass dimension, and $0<\left(M_{1}, W_{F}\right) \ll M_{0}$. These parameters reflect the internal structure of a particle.

The current derivation and understanding of massenergy relation is mainly limited to the Lorentz transformation of parameters of motion, but does not go deep into the dynamical structure of particles. Therefore the relation only reflects the main part, and does not reveal the rich contents. For the following reasons: 1) This is a universal relation obeyed by all matter; 2) It associates with the inner connection between particles and fields; 3) It associates with the relationship between space-time and motion; 4) It associates with classical and quantum opinions; 5) The relation occupies the core of fundamental physics with clear physical meanings. Therefore, how to accurately measure the relation may be a breakthrough to discover new physical phenomena and internal structure of space-time and elementary particles.

\subsection{The Quaternion Form of Other Field Equations}

From the above calculations we learn that, the quaternion form of coordinate increment and spinor equation can simultaneously display coordinates and tetrad, and this form is more convenient for understanding and calculations. In fact, other physical fields also can be expressed in quaternion form.

For vector field with spin $s=1$, we have the first order field equation in quaternion form,

$$
\left\{\begin{array}{l}
\beta^{\mu} \partial_{\mu} A=-\tilde{A}, \\
\tilde{\beta}^{\mu} \partial_{\mu} \tilde{A}=-a^{2} A+e q,
\end{array}\right.
$$

where $q$ is a source term, $\beta^{0}=I$ and

$$
\begin{aligned}
\beta^{1} & =\left(\begin{array}{cccc}
0 & 1 & 0 & 0 \\
1 & 0 & 0 & 0 \\
0 & 0 & 0 & i \\
0 & 0 & -i & 0
\end{array}\right), \beta^{2}=\left(\begin{array}{cccc}
0 & 0 & 1 & 0 \\
0 & 0 & 0 & -i \\
1 & 0 & 0 & 0 \\
0 & i & 0 & 0
\end{array}\right), \\
\beta^{3} & =\left(\begin{array}{cccc}
0 & 0 & 0 & 1 \\
0 & 0 & i & 0 \\
0 & -i & 0 & 0 \\
1 & 0 & 0 & 0
\end{array}\right),
\end{aligned}
$$




$$
\left\{\begin{array}{l}
A=\left(A^{0}, A^{1}, A^{2}, A^{3}\right)^{T}, \\
\tilde{A}=\left(H^{0}, H^{1}, H^{2}, H^{3}\right)^{T}, \\
q=\left(q^{0}, q^{1}, q^{2}, q^{3}\right)^{T}, \\
\tilde{\beta}^{\mu}=\left(\beta^{0},-\beta^{1},-\beta^{2},-\beta^{3}\right) .
\end{array}\right.
$$
have

By current conservation or normalizing condition we

$$
\partial_{\mu} q^{\mu}=0, \quad H^{0}=\partial_{\mu} A^{\mu}=0,
$$

Expanding (4.2), we get the total Maxwell equation system in 3 dimensional vector form,

$$
\begin{cases}\partial_{\mu} q^{\mu}=\partial_{\mu} A^{\mu}=0, & \partial^{\alpha} \partial_{\alpha} A^{\mu}+a^{2} A^{\mu}=e q^{\mu}, \\ \boldsymbol{E}=-\nabla A^{0}-\partial_{0} \boldsymbol{A}, & \boldsymbol{B}=\nabla \times \boldsymbol{A}, \\ \nabla \cdot \boldsymbol{E}=-a^{2} A^{0}+e q^{0}, & \nabla \times \boldsymbol{E}=-\partial_{0} \boldsymbol{B}, \\ \nabla \cdot \boldsymbol{B}=0, & \nabla \times \boldsymbol{B}=\partial_{0} \boldsymbol{E}-a^{2} \boldsymbol{A}+e \boldsymbol{q},\end{cases}
$$

in which

$$
\boldsymbol{H} \equiv \boldsymbol{E}+\boldsymbol{B} i, \quad \boldsymbol{A} \equiv\left(A^{1}, A^{2}, A^{3}\right), \quad \boldsymbol{q} \equiv\left(q^{1}, q^{2}, q^{3}\right) .
$$

For long distance interaction such as electromagnetic field, the distance factor $a=0$ in (4.6). From (4.2), we find magnetic charge should be the imaginary part of electric charge. Unfortunately, this part leads to the violation of normalizing condition, that is to say, the charge conservation is broken. This is absurd, so the monopole can not exist in the nature.

The strong interaction also should be described by (4.2). In this case, we should have $a>0$.

The transformation law of the complex field $\tilde{A}$ is interesting. Make boosting transformation along $X^{1}$, we have Lorentz transformation $X^{\prime}=\Lambda X$,

$$
\Lambda(\xi)=\operatorname{diag}\left[\left(\begin{array}{cc}
\cosh \xi & -\sinh \xi \\
-\sinh \xi & \cosh \xi
\end{array}\right), 1,1\right] .
$$

Then the transformation of $\tilde{A}$ becomes $\tilde{A}^{\prime}=\tilde{\Lambda} \tilde{A}$, where

$$
\tilde{\Lambda}=\operatorname{diag}\left[1,1,\left(\begin{array}{cc}
\cosh \xi & i \sinh \xi \\
-i \sinh \xi & \cosh \xi
\end{array}\right)\right]
$$

When making space rotation transformation, $\tilde{A}$ has the same transformation law of coordinate system, that is $\tilde{\Lambda}=\Lambda$.

Similar to (4.2), for tensor field with spin $s=2$, we have dynamics

$$
\left\{\begin{array}{l}
\beta^{\mu} \partial_{\mu} G=\tilde{G} \\
\tilde{\beta}^{\mu} \partial_{\mu} \tilde{G}=\lambda G+\kappa T
\end{array}\right.
$$

in which $G=\left(G^{\mu v}\right)$ is contravariant tensor in matrix form, $\tilde{G}$ is the complex field intensity, and $T$ is source term.

Obviously, (3.2), (4.2) and (4.10) all take the form of (2.5). Almost all known physical laws and relations can be derived from some combination of these equations, so (2.5) is the most fundamental and universal law of physics, and it actually forms a kind of unified field theory.

In (4.2) and (4.10), the components of fields are projected to the orthogonal tetrad. How to transform the equation into curved space-time and establish the relation between the components is still an open problem. Of course, the transformation is just a choice of description. We can make deep understanding into the structure of space-time and fields, and find new internal symmetries. However, like the chiral transformation, the transformation can not produce new physical phenomenon, because the physical phenomenon is objective, but the transformation is subjective.

\section{Conclusions}

From the above specific discussion and calculation, we can get the following conclusions:

1) The space-time has a quaternion structure, which should be naturally described by the basic relations (2.1)(2.4).

2) The evolution of a given physical system is governed by the universal dynamical Equation (2.5), and in principle, all properties of the system can be logically derived from the dynamical equation together with the concrete initial and boundary conditions. The solution of the first order dynamics (2.5) determines the arrow of time, and the non-uniqueness of evolution of spinor fields breaks the reversibility of the process.

3) The invariance and covariance of the dynamics are different concepts from the symmetry of the solutions, although there are some connections between them. The physical laws must be invariant and covariant, but the solutions or the configurations of concrete matter system are not definitely symmetric.

\section{REFERENCES}

[1] S. F. Savitt, "Time's Arrows Today," Cambridge University Press, Cambridge, 1995. doi:10.1017/CBO9780511622861

[2] J. L. Lebowitz, "Boltzmann's Entropy and Time's Arrow," Physics Today, Vol. 46, No. 9, 1993, pp. 32-38.

[3] P. M. Harman, "The Natural Philosophy of James Clerk Maxwell," Cambridge University Press, Cambridge, 1998.

[4] D. Ruelle, "Chance and Chaos," Princeton University Press, New Jersey, 1991.

[5] D. Albert, "Time and Chance," Harvard University Press, Cambridge, 2000. 
[6] H. D. Zeh, "The Physical Basis of the Direction of Time," Springer-Verlag, Berlin, 2001.

[7] S. W. Hawking, R. Laflamme and G. W. Lyons, "The Origin of Time Asymmetry," arXiv:gr-qc/9301017.

[8] J. W. Moffat, "Quantum Gravity, the Origin of Time and Time's Arrow," arXiv:gr-qc/9209001.

[9] I. Dincer and Y. A. Cengel, "Energy, Entropy and Exergy Concepts and Their Roles in Thermal Engineering," Entropy, Vol. 3, No. 3, 2001, pp. 116-149. doi:10.3390/e3030116

[10] G. F. R. Ellis, “On the Flow of Time," arXiv:0812.0240.

[11] Y. Q. Gu, "Some Subtle Concepts in Fundamental Physics," arXiv:0901.0309v1

[12] Y. Q. Gu, "Some Paradoxes in Special Relativity and the Resolutions," Advances in Applied Clifford Algebras, Vol. 21, No. 1, 2011, pp. 103-119. doi:10.1007/s00006-010-0244-6

[13] G. F. R. Ellis and J.-Ph. Uzan, "c Is the Speed of Light, Isn't It?" American Journal of Physics, Vol. 73, No. 3, 2005, pp. 240-247. doi:10.1119/1.1819929

[14] Y. Q. Gu, "The Vierbein Formalism and Energy-Momentum Tensor of Spinors," arXiv:gr-qc/0612106.
[15] Y. Q. Gu, "The Spinor Connection and Its Dynamical Effects," arXiv:gr-qc/0610001v3.

[16] Y. Q. Gu, "A Canonical Form for Relativistic Dynamic Equation," Advances in Applied Clifford Algebras, Vol. 7, No. 1, 1997, pp. 13-24. doi:10.1007/BF03041212

[17] G. F. R. Ellis, "On the Limits of Quantum Theory: Contextuality and the Quantum-Classical Cut," arXiv:1108.5261.

[18] OPERA Collaboration, "Measurement of the Neutrino Velocity with the OPERA Detector in the CNGS Beam," arXiv: $1109.4897 \mathrm{v} 2$.

[19] Y. Q. Gu, "Some Properties of the Spinor Soliton," Advances in Applied Clifford Algebras, Vol. 8, No. 1, 1998, pp. 17-29. doi:10.1007/BF03041923

[20] Y. Q. Gu, "The Characteristic Functions and Their Typical Values for the Nonlinear Spinors," arXiv:hep-th/0611210.

[21] Y. Q. Gu, "New Approach to N-body Relativistic Quantum Mechanics," International Journal of Modern Physics, Vol. A22, 2007, pp. 2007-2020. doi:10.1142/S0217751X07036233

[22] Y. Q. Gu, “A Sensitive Test of Mass-Energy Relation," arXiv:hep-th/0610189. 